\title{
DUN-525 13
}

\section{Cover Sheet for a Hanford Historical Document Released for Public Availability}

Released 1994

Prepared for the U.S. Department of Energy under Contract DE-AC06-76RLO 1830

Pacific Northwest Laboratory Operated for the U.S. Department of Energy by Battelle Memorial Institute

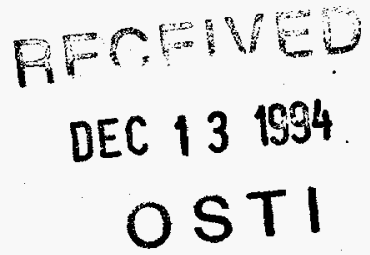




\section{DISCLAIMER}

This report was prepared as an account of work sponsored by an agency of the United States Government. Neither the United States Government nor any agency thereof, nor any of their employees, make any warranty, express or implied, or assumes any legal liability or responsibility for the accuracy, completeness, or usefulness of any information, apparatus, product, or process disclosed, or represents that its use would not infringe privately owned rights. Reference herein to any specific commercial product, process, or service by trade name, trademark, manufacturer, or otherwise does not necessarily constitute or imply its endorsement, recommendation, or favoring by the United States Government or any agency thereof. The views and opinions of authors expressed herein do not necessarily state or reflect those of the United States Government or any agency thereof. 


\section{DISCLAIMER}

Portions of this document may be illegible in electronic image products. Images are produced from the best available original document. 


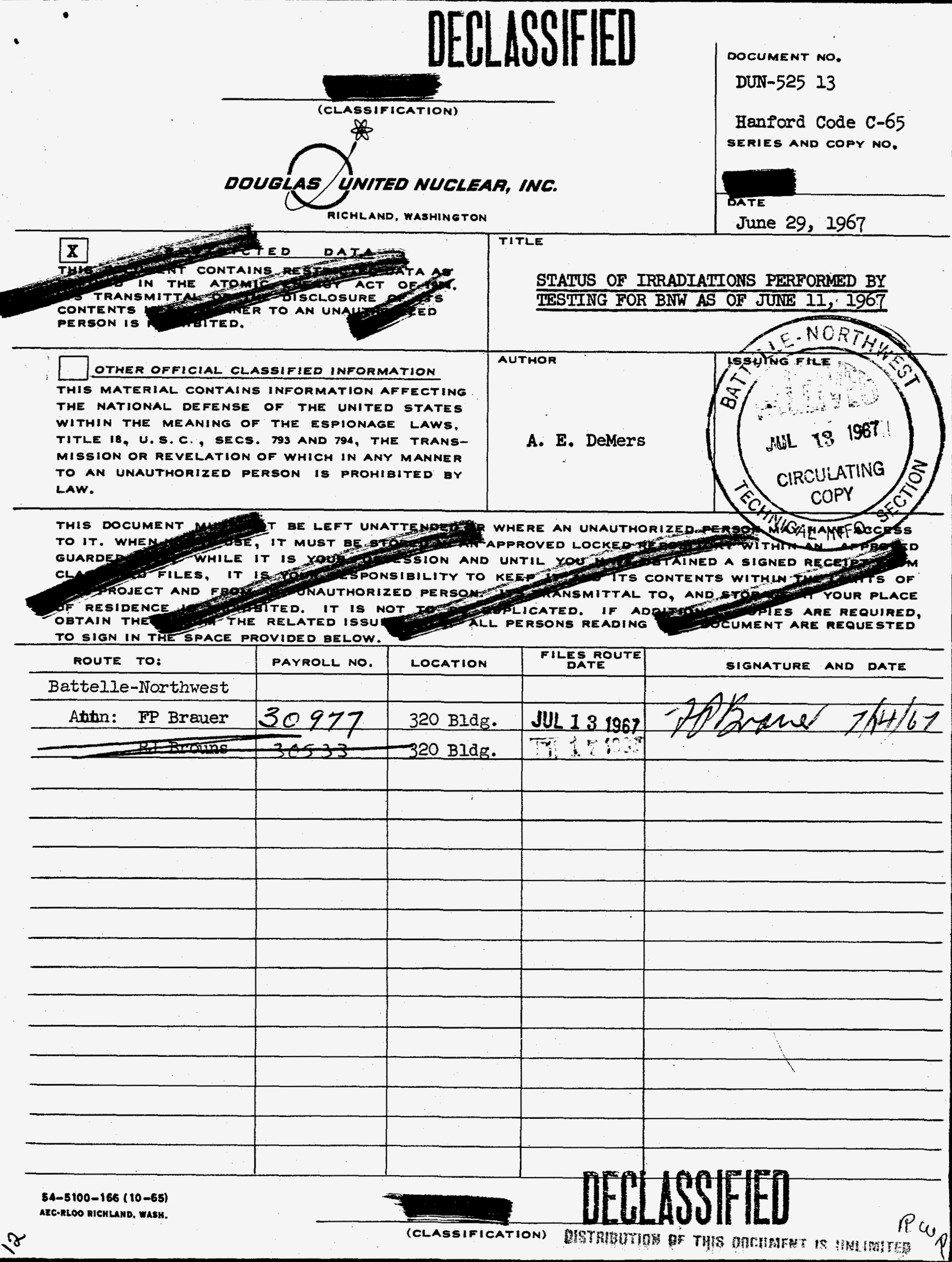




\section{DECLLASSIFED}

This document classified by:

DUN-525 13

$6 / 29 / 67$

Hanford Code C-65

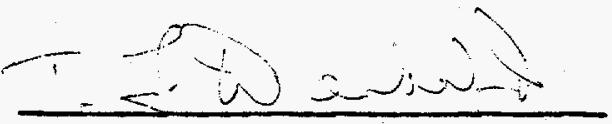

This document consists of 11 pages

\section{DISTRIBUTION}

1. AE DeMers

2. DUN File

3. DUN Record

Battelle-Northwest

4. FP Breuer/RJ Brouns

5. TT Claudson

6. AJ Jervis/JH Cox

7. RD Leggett

STATUS OF IRRADIATIONS PERFORMED BY

TESTING FOR BNW AS OF JUNE 11, 1967

A. E. DeMers

Clasuification Cancolled and Chrooed to

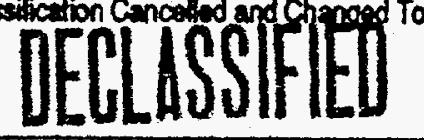

By Authoriy of RHelten

$C G-N M P-1,8-3-94$

By Es Savely 9-20-94

variod By Gerri Maley 9-21-94.

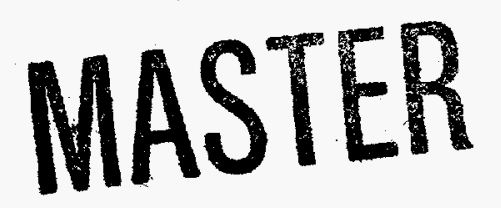


This report itemizes the irradiations performed by Testing for BattelleNorthwest. It lists the material being irradiated, awaiting disposition and material shipped during the report period. An explanation of the various columns in the report follows:

Identification

a. TISR No. - This is the officiel number assigned by Testing to an experiment for purposes of identification.

b. Request Ihumber - Customer experiment identification number, if any.

c. Material - The principle target material, if known, abbreviated or Its chemical symbol in capital letters.

d. Piece Number - Individual sample or assembly identification.

Operating Time (days) - The number of full power operating days the sample has been exposed.

CMK Absorbed - The total cmk absorbed by the sample. Does not include the facility.

Charge Date - Date and time sample charged into reactor or date and time reactor reached one-half equilibrium level if sample was charged during a reactor outage.

Location

a. Facility - Test facility or process tube and reactor containing the experiment.

b. Test Hole Position - Sample position in reactor relative to a process tube.

c. Position Factor - Location of sample in relation to front-to-rear cosine flux distribution. (This factor only applies to front-to-rear irradiations.)

NOTE: The actual flux shape may deviate from a cosine curve due to local perturbations. The actual shape will be provided upon request.

Exposure to Date - NVT

The total thermal neutron exposure per square centimeter received to date This number is calculated from facility unperturbed flux and reactor operating time.

Discharge, Date and Time

Date and time samples were removed from the active portion of the reactor or date and time reactor was down to one-half equilibrium level if sample was discharged during a reactor outage. 
DUN -52513

\section{Shipping Date}

Date samples were shipped to the customer or otherwise disposed of.

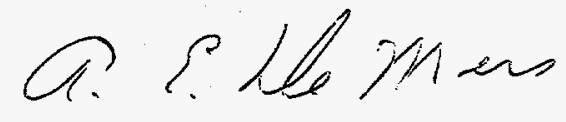

\section{Acting Supervisor}

Irradiation Testing

AE DeMers/kh

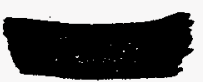




\begin{tabular}{|c|c|c|c|c|c|c|c|c|c|c|c|c|c|c|}
\hline \multicolumn{4}{|c|}{ IDENTIFICATION } & \multirow{2}{*}{$\begin{array}{l}\text { OPERATING } \\
\text { TIME } \\
\text { (DAYS) }\end{array}$} & \multirow{2}{*}{$\begin{array}{c}\text { C M K } \\
\text { ABSORBED }\end{array}$} & \multicolumn{2}{|l|}{ CHARGE } & \multicolumn{3}{|c|}{ LOCATION } & \multirow{2}{*}{$\begin{array}{c}\text { EXPOSURE } \\
\text { TODATE } \\
\text { NVT }\end{array}$} & \multicolumn{2}{|c|}{ DISCHARGE } & \multirow{2}{*}{$\begin{array}{c}\text { SHIPPING } \\
\text { DATE }\end{array}$} \\
\hline $\begin{array}{l}\text { TISR } \\
\text { No. }\end{array}$ & $\begin{array}{l}\text { REOUEST } \\
\text { NUMBER }\end{array}$ & MATERIAL & $\begin{array}{l}\text { PIECE } \\
\text { NO. }\end{array}$ & & & DATE & TIME & FACLLITY & $\begin{array}{l}\text { TEST HOLE } \\
\text { POSITION }\end{array}$ & $\begin{array}{l}\text { POSITION } \\
\text { FACTOR }\end{array}$ & & DATE & TIME & \\
\hline $\begin{array}{l}T H \\
177 \\
177 \\
177 \\
177 \\
177 \\
177 \\
177 \\
177 \\
177 \\
177 \\
177 \\
177 \\
177 \\
177 \\
177 \\
177 \\
177 \\
177 \\
177 \\
177 \\
177 \\
222 \\
222 \\
222 \\
236 \\
236 \\
236 \\
243 \\
243 \\
243 \\
243 \\
243 \\
259 \\
259 \\
295 \\
307 \\
372 \\
372\end{array}$ & $=$ & 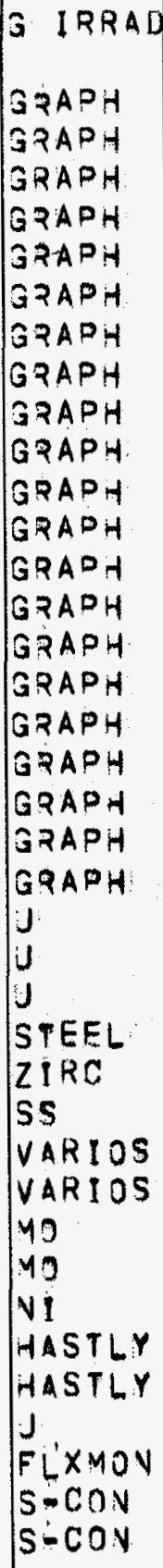 & 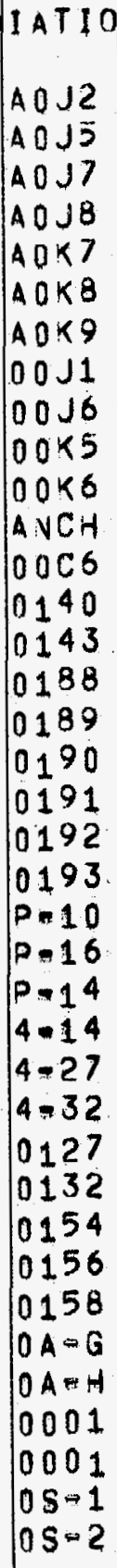 & $\begin{array}{r}S A R=I N P \\
206.9872 \\
131.1053 \\
131.1053 \\
131.1053 \\
131.1053 \\
131.1053 \\
206.9872 \\
37.4458 \\
37.4458 \\
37.4458 \\
37.4458 \\
290.1917 \\
280.1917 \\
280.1917 \\
290.1917 \\
280.1917 \\
280.1917 \\
280.1917 \\
290.1917 \\
280.1917 \\
280.1917 \\
33.6993 \\
93.1707 \\
11.2958 \\
535.8943 \\
11.2958 \\
32.0443 \\
223.8898 \\
193.2898 \\
13.3020 \\
13.3020 \\
13.3020 \\
645.3950 \\
645.3950 \\
958.5452 \\
691.8943 \\
2.9736 \\
2.9736\end{array}$ & PROGRE & 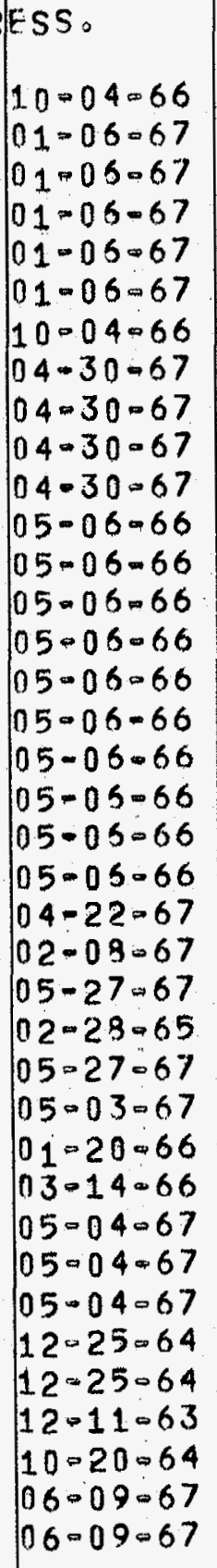 & 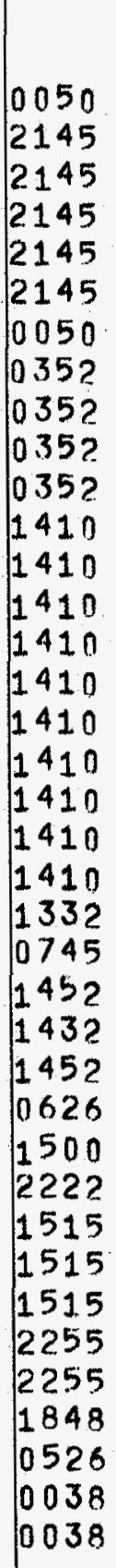 & 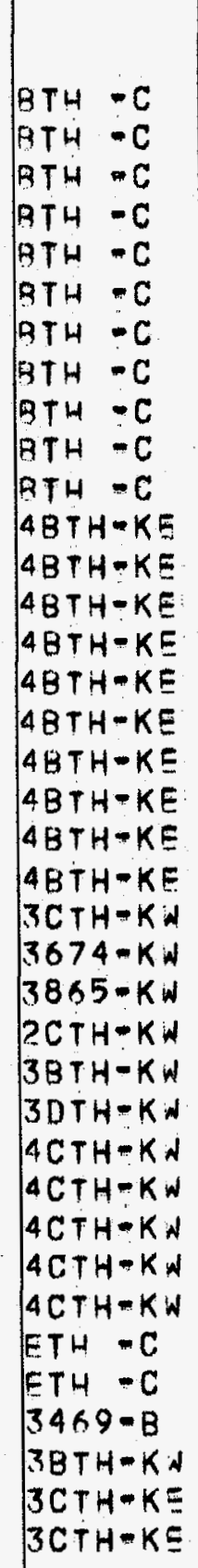 & $\begin{array}{l}0-2089 \\
0-2074 \\
0-2077 \\
0-2065 \\
0-2080 \\
0-2083 \\
0-2086 \\
0-2062 \\
0-2071 \\
0-2059 \\
0-2068 \\
0-4382 \\
0-4367 \\
0-4379 \\
0-4376 \\
0-4364 \\
0-4361 \\
0-4373 \\
0-4370 \\
0-4358 \\
0-4355\end{array}$ & 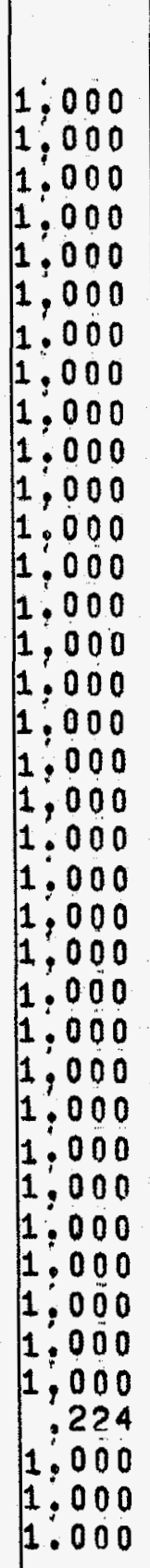 & 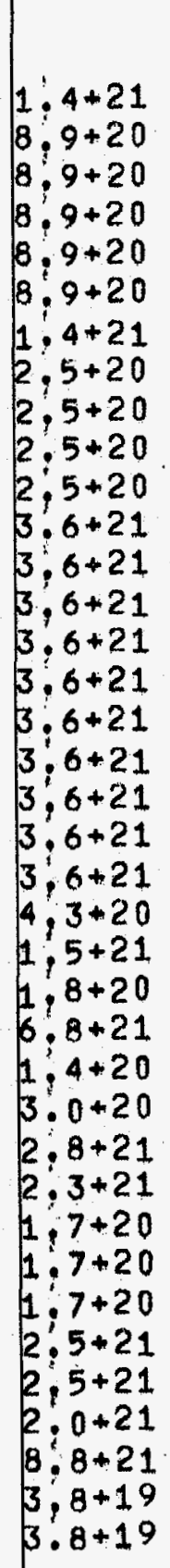 & & & - \\
\hline
\end{tabular}




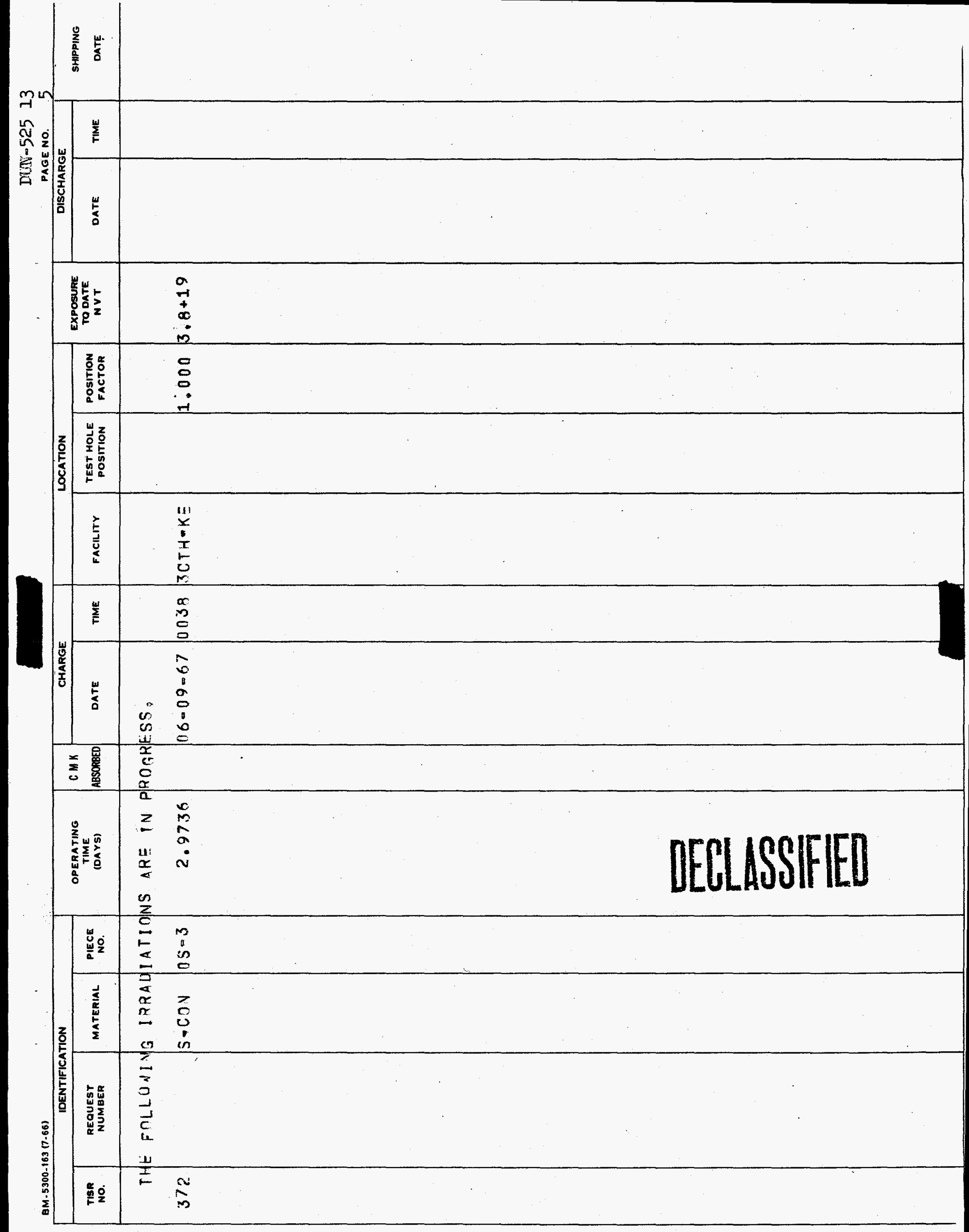




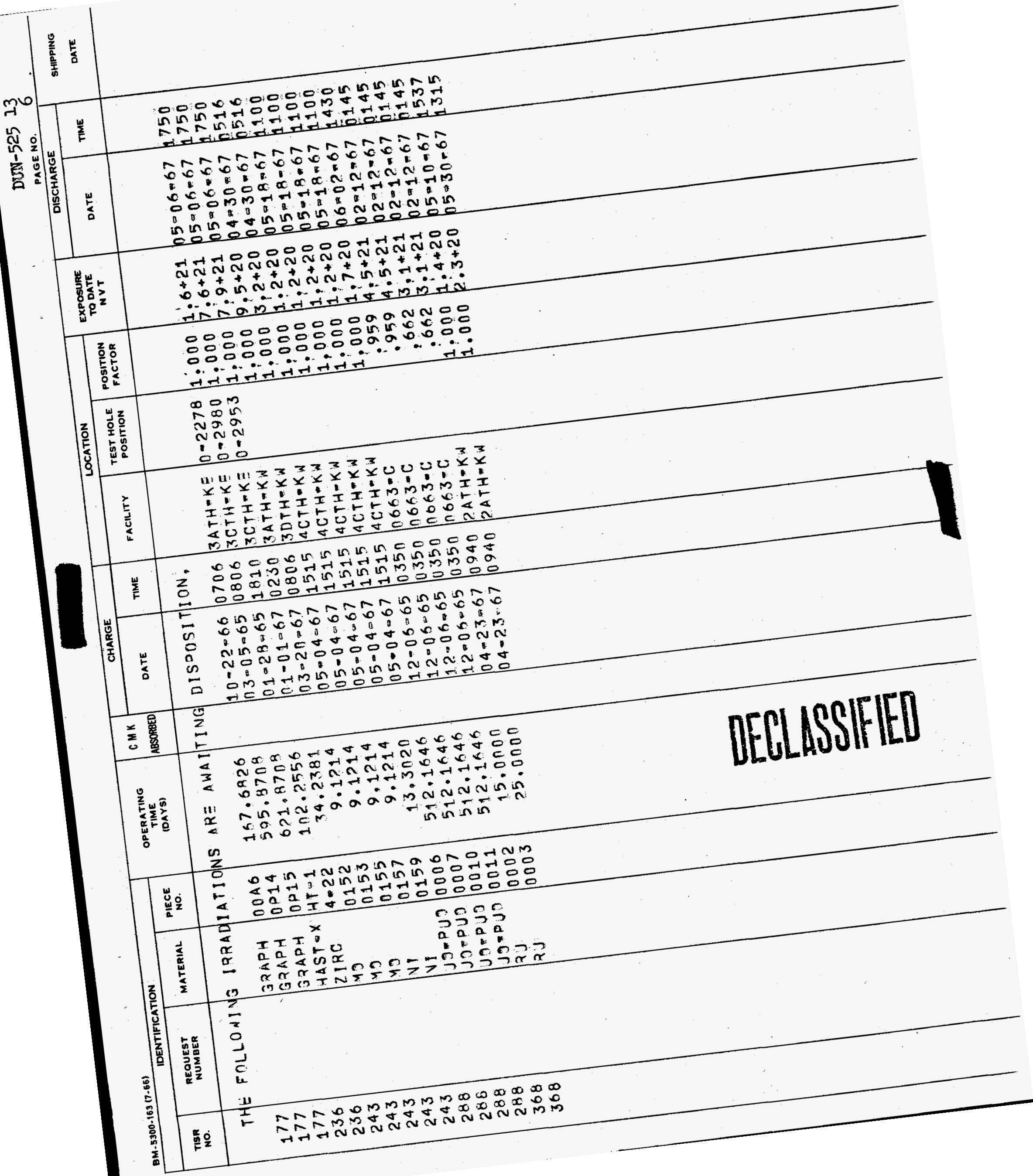




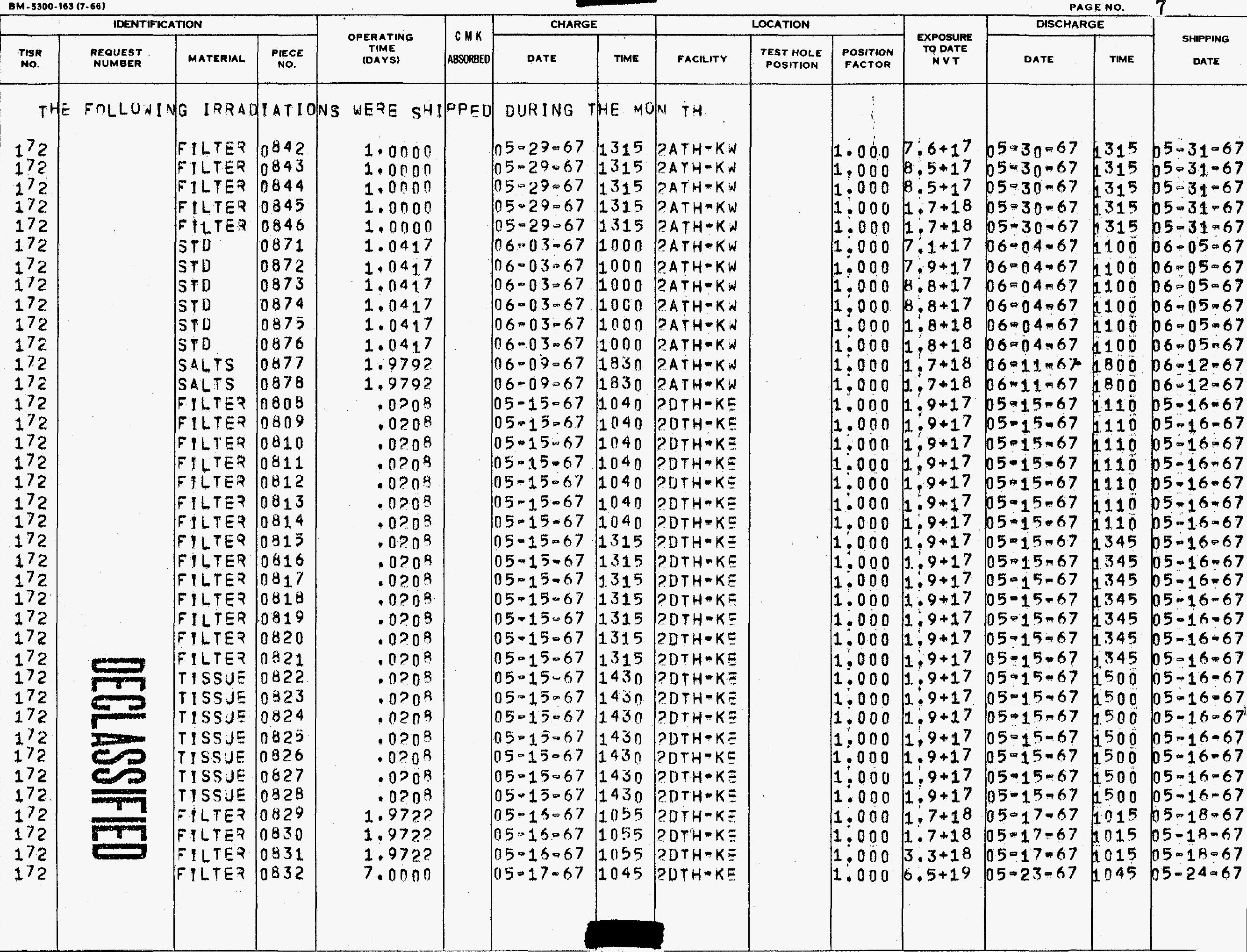




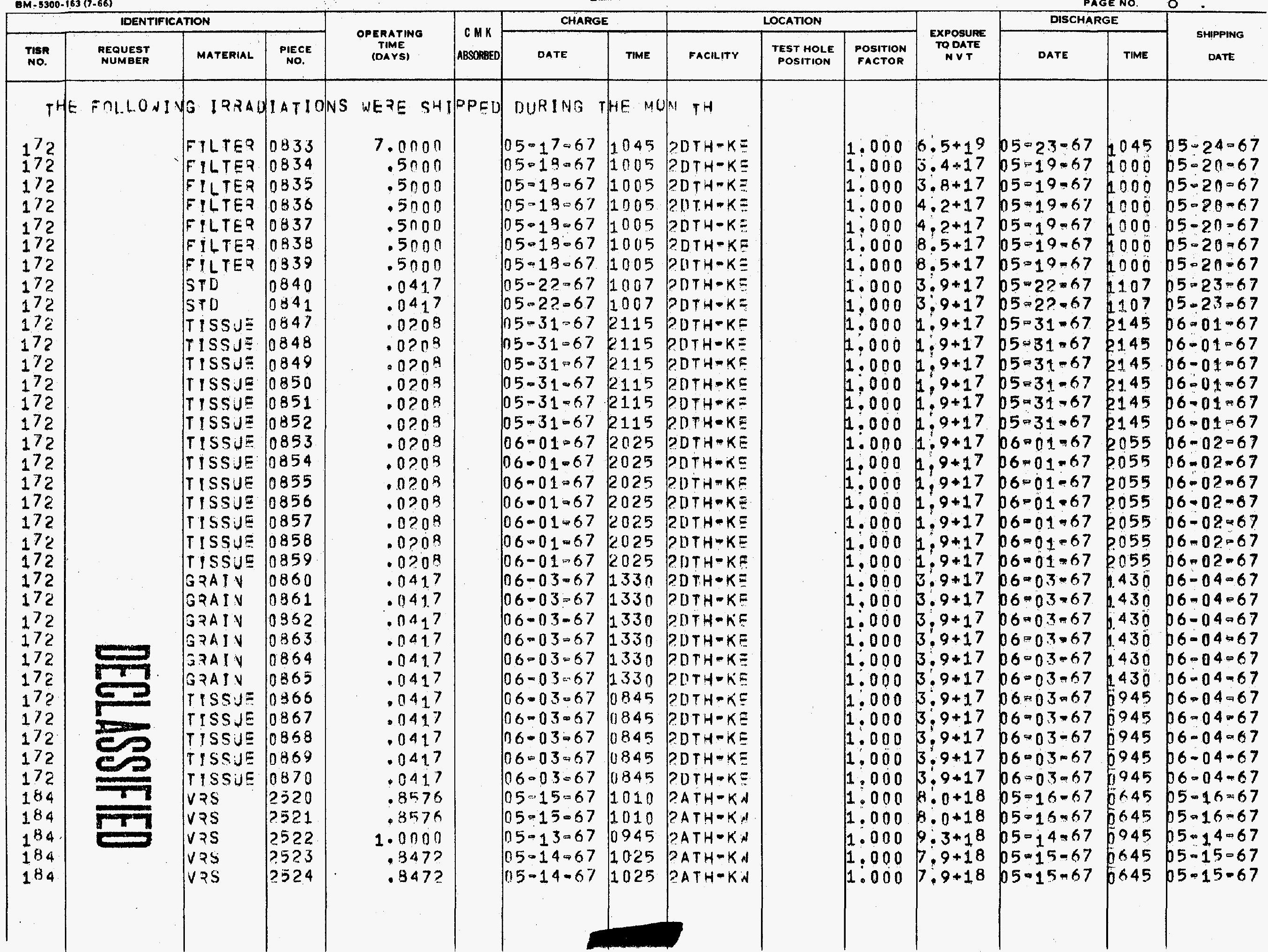




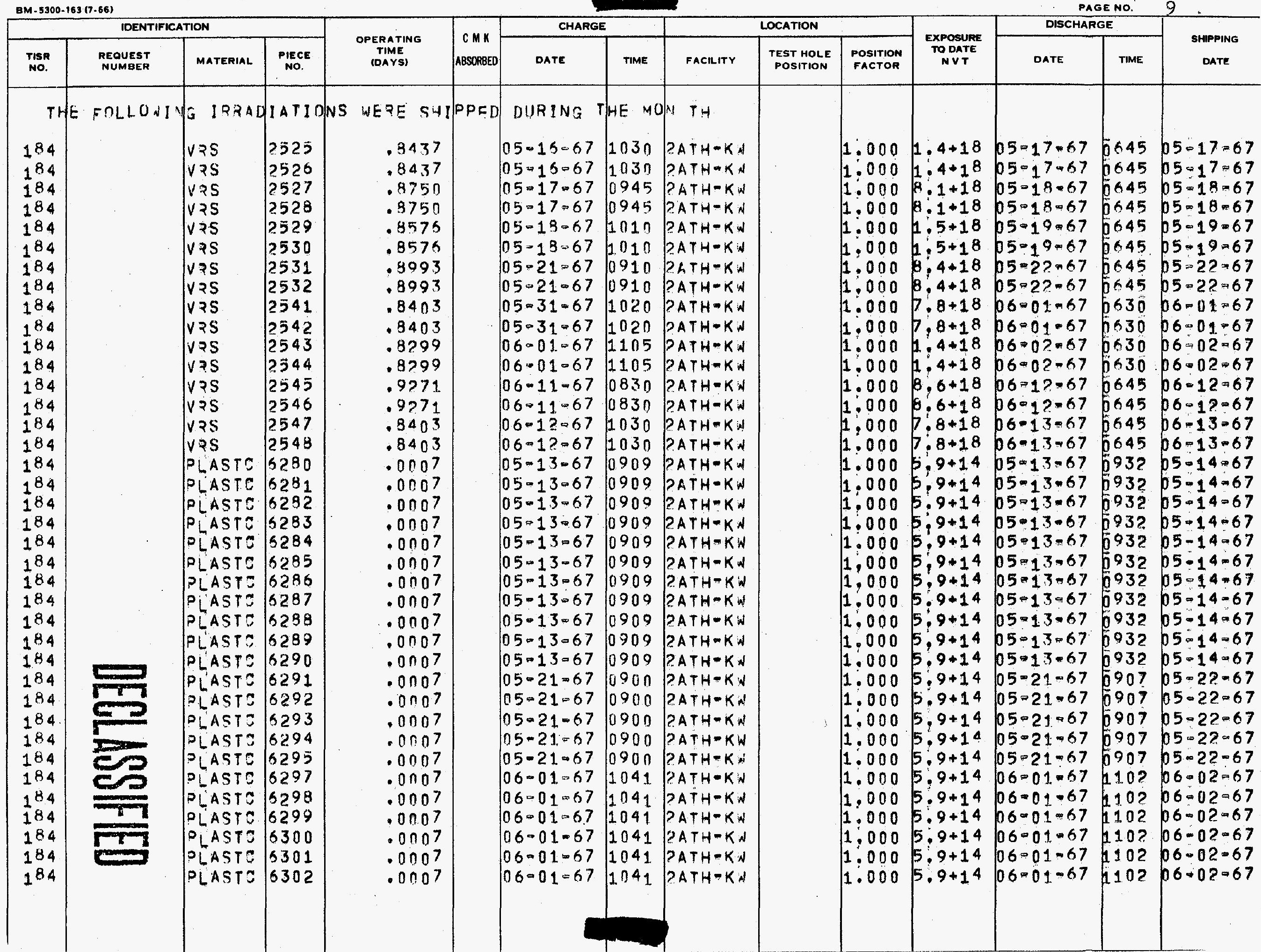




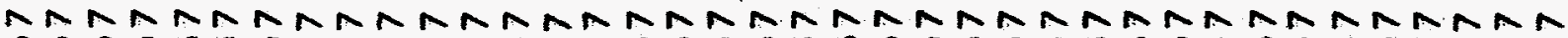

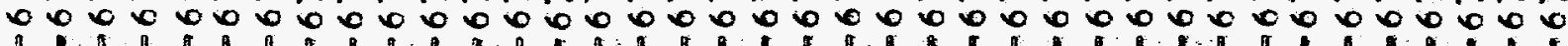

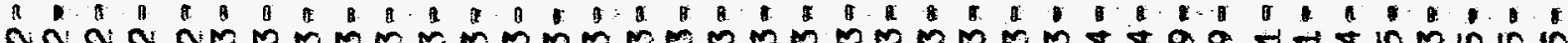

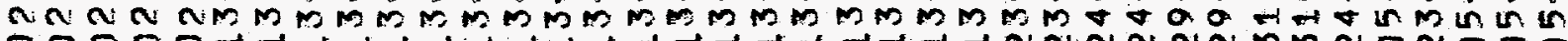

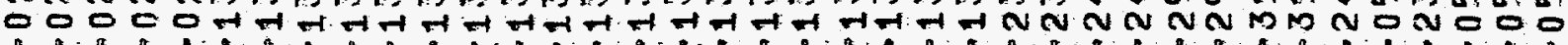

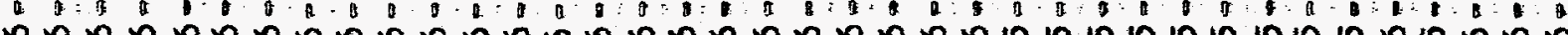

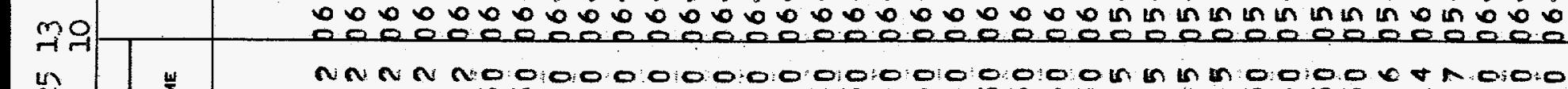

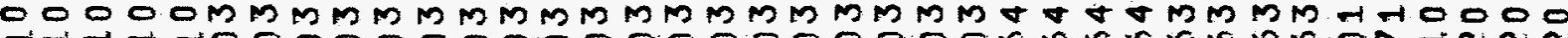

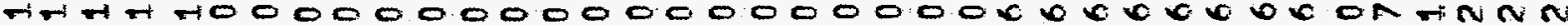

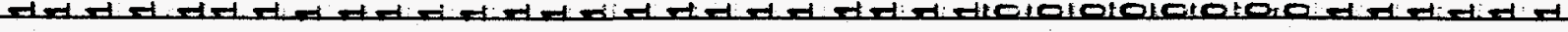

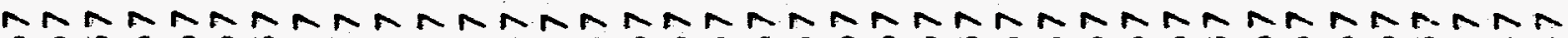

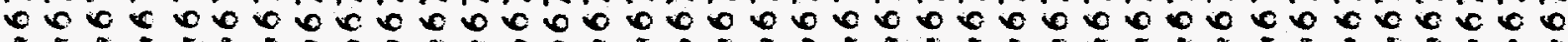

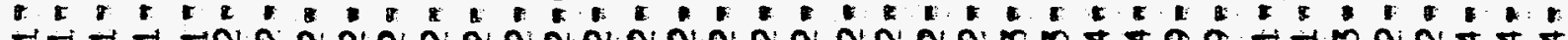
HHH HNON

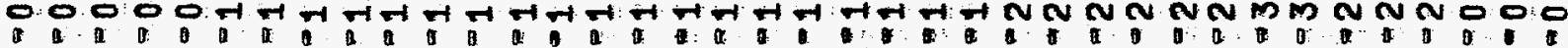
06000000000000000000000

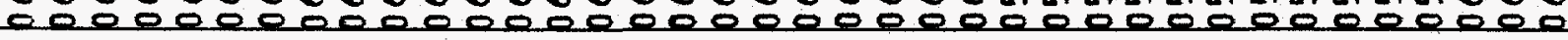

\begin{tabular}{|c|}
\hline 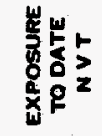 \\
\hline
\end{tabular}

¿\&

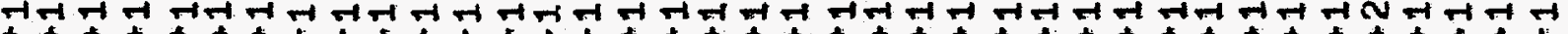

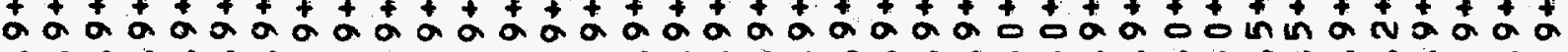

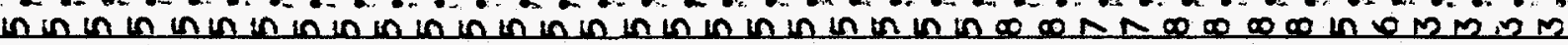

\begin{tabular}{|c|c|}
\hline 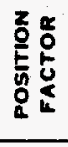 & 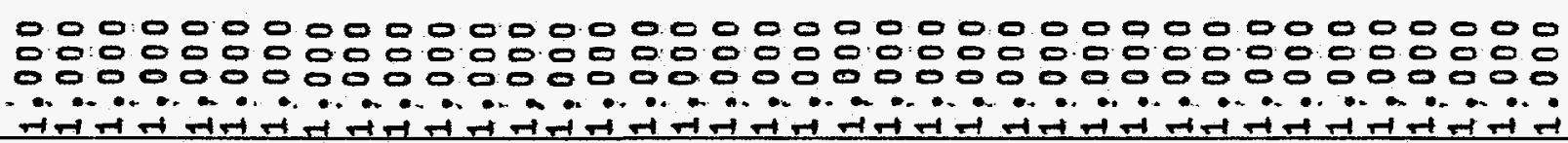 \\
\hline
\end{tabular}

\section{zo}

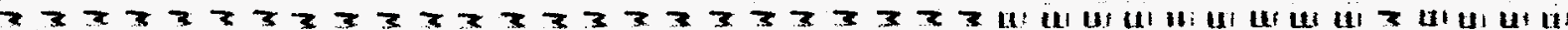

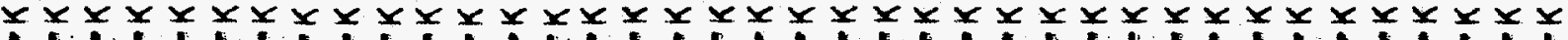

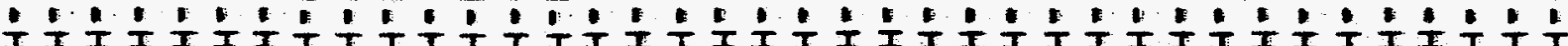
IIIIIIIIIIIIIIIIIIIIIIIIIIIIIIIIIIIIIIIII

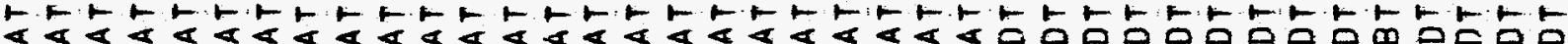

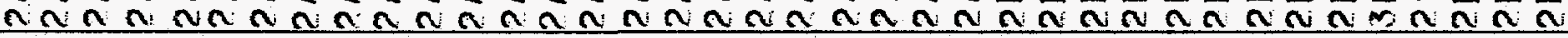

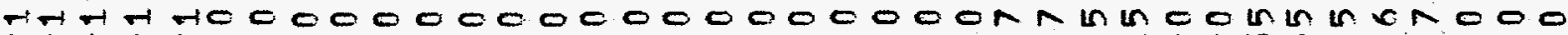
* J J Jo00000000000000000000 Haninmm $00000000000000000000000000000000000 \%$

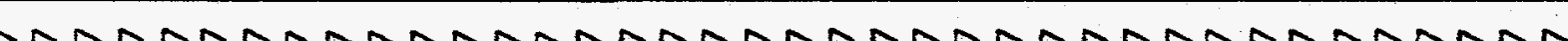
두

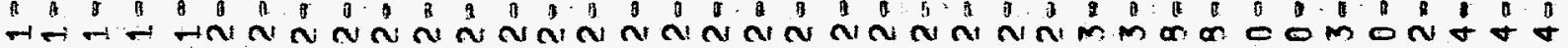

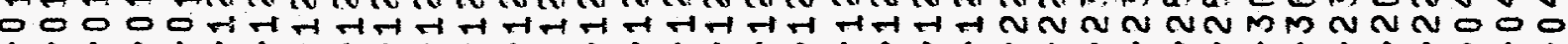
1

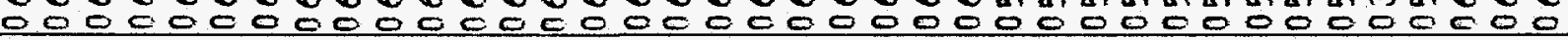

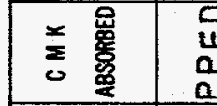

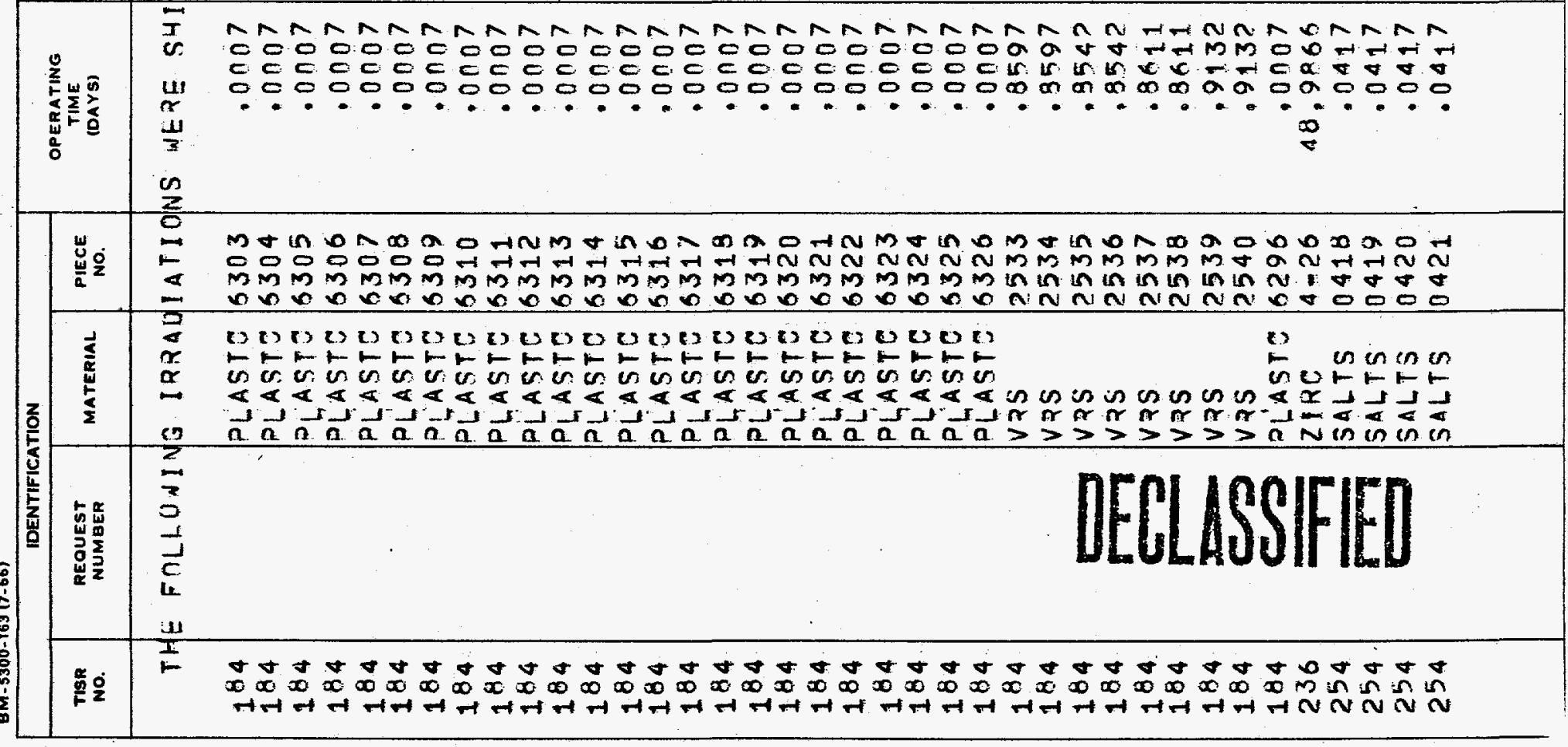




\begin{tabular}{|c|c|c|c|c|}
\hline \multirow{3}{*}{ 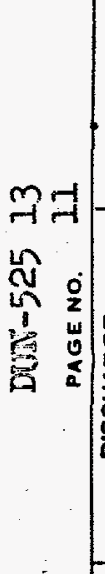 } & \multicolumn{2}{|c|}{ 亮 } & & 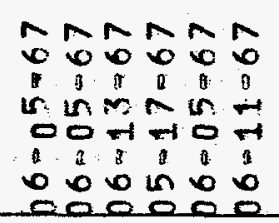 \\
\hline & \multirow{2}{*}{ 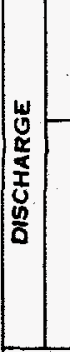 } & $\sum_{\underline{F}}^{\frac{\mathrm{w}}{2}}$ & & 이에 \\
\hline & & $\frac{\omega}{a}$ & & 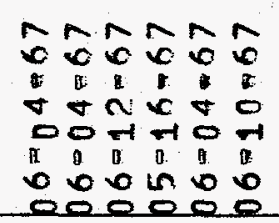 \\
\hline & \multicolumn{2}{|c|}{ 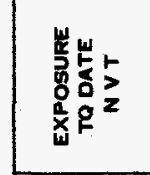 } & & 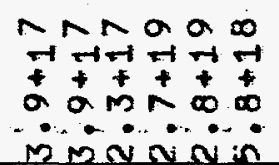 \\
\hline & \multirow{3}{*}{ 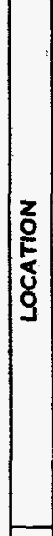 } & $\begin{array}{l}2 \\
0 \\
0 \\
0 \\
0 \\
0 \\
0 \\
0 \\
0\end{array}$ & & 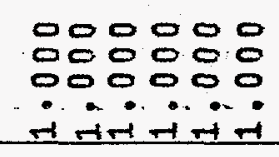 \\
\hline & & 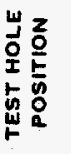 & & \\
\hline & & 站 & $\begin{array}{l}x \\
z \\
z\end{array}$ & 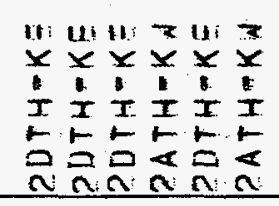 \\
\hline & \multirow[b]{2}{*}{ 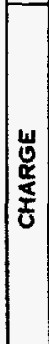 } & $\sum_{F}^{\frac{\omega}{F}}$ & W & 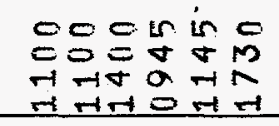 \\
\hline & & 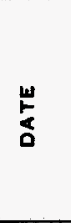 & 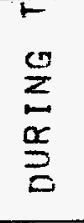 & 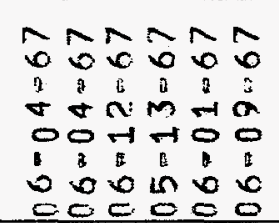 \\
\hline & $\underset{\Sigma}{\Sigma}$ & 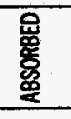 & $\begin{array}{l}\frac{c}{u} \\
\frac{a}{a} \\
\end{array}$ & \\
\hline & 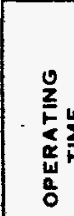 & 岁高 & $\begin{array}{l}\vec{J} \\
u \\
u \\
\vec{\sigma} \\
\vec{w}\end{array}$ & 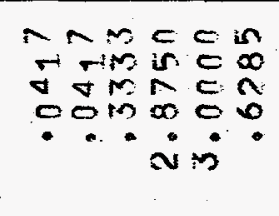 \\
\hline & \multirow{4}{*}{ 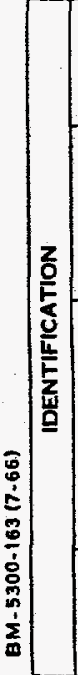 } & 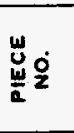 & 5 & 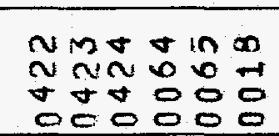 \\
\hline & & 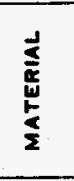 & $\begin{array}{l}a \\
\pi \\
a \\
0 \\
0\end{array}$ & 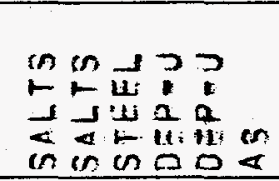 \\
\hline & & 留离 & 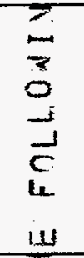 & \\
\hline 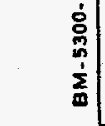 & & $\frac{\alpha}{2} \frac{\dot{\alpha}}{2}$ & \pm & 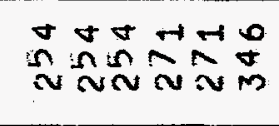 \\
\hline
\end{tabular}

